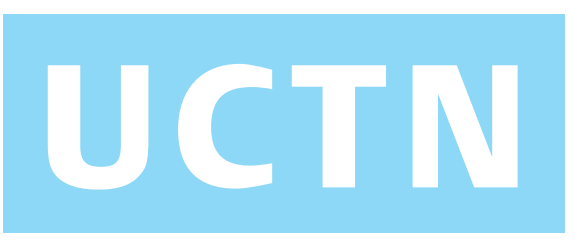

\title{
Traumatic Disruption of the Proximal Hepatic Duct: Endoscopic Treatment After Failure of Surgical Repair
}

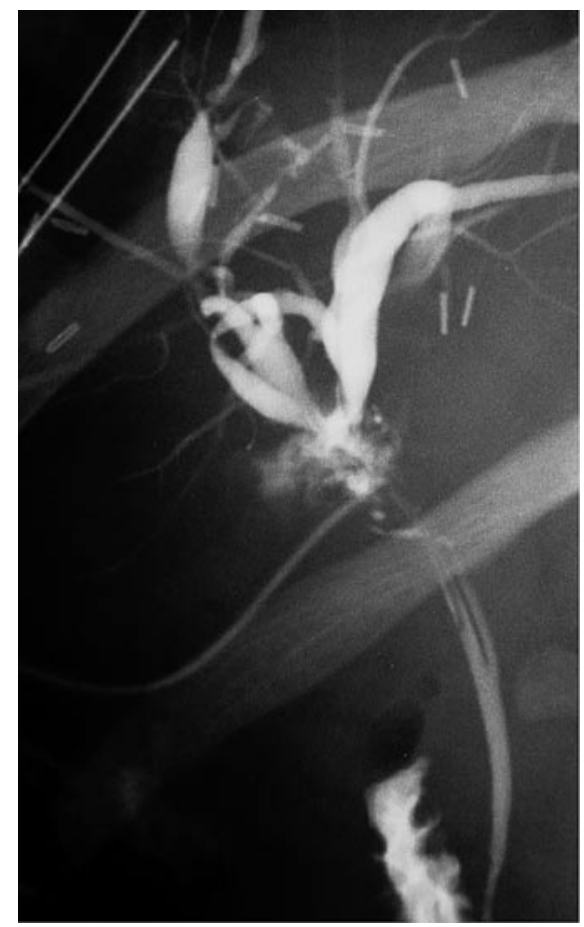

Figure 1 Cholangiography via T-tube drainage at POD 4 shows an extravasation of contrast medium at the end-to-end anastomosis of the proximal common bile duct.

We describe the successful endoscopic treatment of a patient with nearly complete disruption of the proximal common hepatic duct. A 19-year-old man, as a front-seat passenger wearing a seat-belt, was involved in a motor vehicle accident in December 1998. Laparotomy revealed a rupture of segments V to VIII, with an extended injury of the right hepatic vein and a deep laceration of segment IV to the hepatic hilus with a tangential lesion of the proximal common hepatic duct involving $25 \%$ of the circumference. Right hemihepatectomy and suture repair of the bile duct was performed. Re-operation was required at day 4 because of a major anastomotic leakage, and 2 days later the patient was referred to our institution because of recurrent bile leakage (Figure 1). Endoscopic retrograde cholangiopancreatography (ERCP) showed almost complete disruption of the proximal hepatic duct. The T-tube was removed

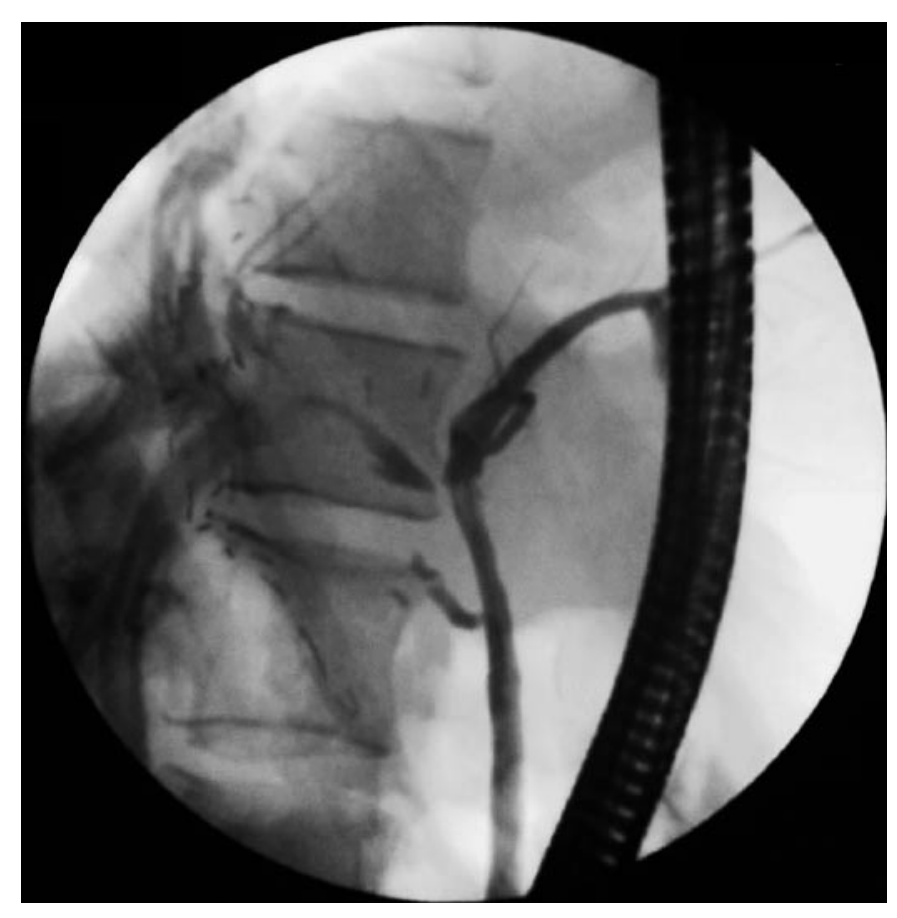

Figure 2 Endoscopic retrograde cholangiopancreatography (ERCP) after injury: A 10-F $10-\mathrm{cm}$ stent is placed over the lesion of the traumatic defect.

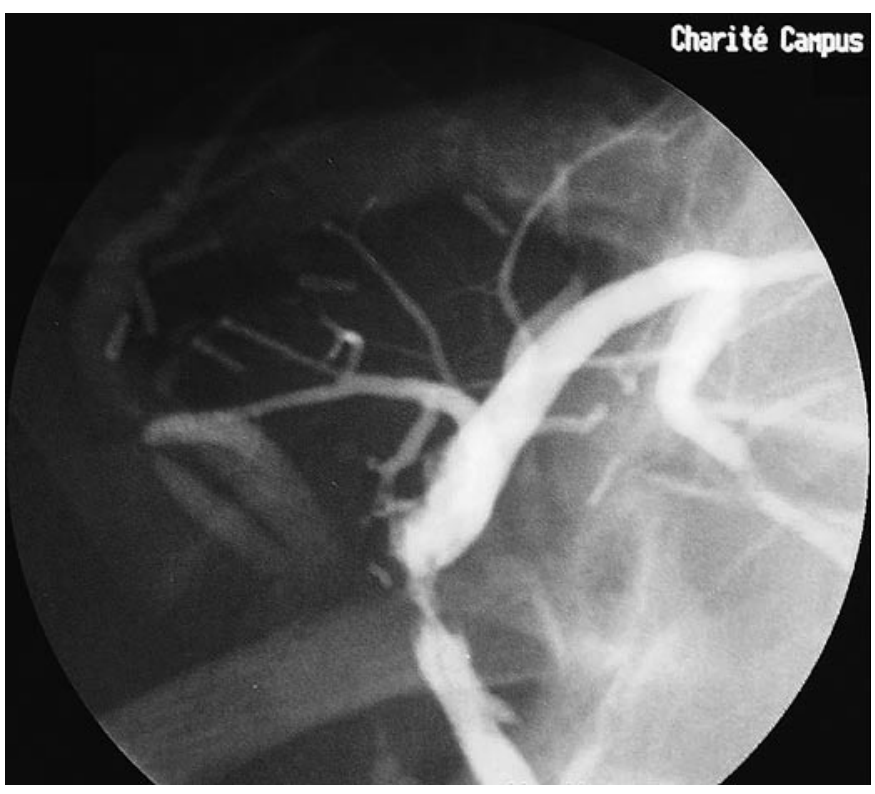

Figure 3 ERCP at 8 months shows a high-grade stricture near the hepatic bifurcation.

and a $10-\mathrm{F} 10-\mathrm{cm}$ stent was inserted over the lesion of the defect (Figure 2). ERCP at 8 months after injury demonstrated a high-grade biliary stricture at the proximal common bile duct (Figure 3). Endobiliary stenting and repeated balloon dilation procedures were carried out with ex- change of the stent every 2 months during the following year. After that year had passed, follow-up ERCP showed no functionally relevant narrowing at the anastomosis. The stent was removed. We continued balloon dilation of the bile duct at 2month intervals for a second year. After 
4.5 years, the patient is well without any clinical or laboratory signs of recurrent stricture (Figure 4).

Small bile duct strictures can be treated by endoscopic options in most patients [1]. Lateral lesions with a diameter less than $5 \mathrm{~mm}$ also can be successfully treated by endoscopic stenting [2]. Lesions of greater than $50 \%$ of the circumference and complete transsections are best managed by bilioenteric anastomosis [3]. In the patient described here, an almost complete disruption of the proximal bile duct was successfully managed by endoscopic stent placement without operative reapproximation of the edges. Currently, nonsurgical interventional procedures, such as endoscopic dilation with stents, can be considered as the primary option in the treatment of patients with posttraumatic bile duct strictures [3]. With good long-term outcomes in $74 \%$ to $90 \%$ of cases after endoscopic treatment, the results are similar to those for surgical repair [4]. Although it is not known which endoscopic procedure in patients with postoperative or post-traumatic strictures is optimal, we recommend the insertion of three $10 \mathrm{~F}$ to $12 \mathrm{~F}$ stents, with a side-to-side technique and which are not exchanged for a total of at least 12 months [5].

\section{S. C. Schmidt ${ }^{1}$, A. Adler ${ }^{2}$, H. Keck ${ }^{3}$,} W. Veltzke-Schlieker ${ }^{2}$, R. E. Hintze ${ }^{2}$, J. M. Langrehr ${ }^{1}$

${ }^{1}$ Department of General, Visceral- and Transplantation Surgery, Charité Campus Virchow Clinic, University Medicine Berlin, Germany

2 Department of Internal Medicine, Division of Hepatology, Gastroenterology, Endocrinology, and Metabolism, Charité Campus Virchow Clinic, Humboldt University of Berlin, Germany ${ }^{3}$ Department of Surgery, Städtisches Klinikum Wolfenbüttel/GgmbH, Wolfenbüttel, Germany.

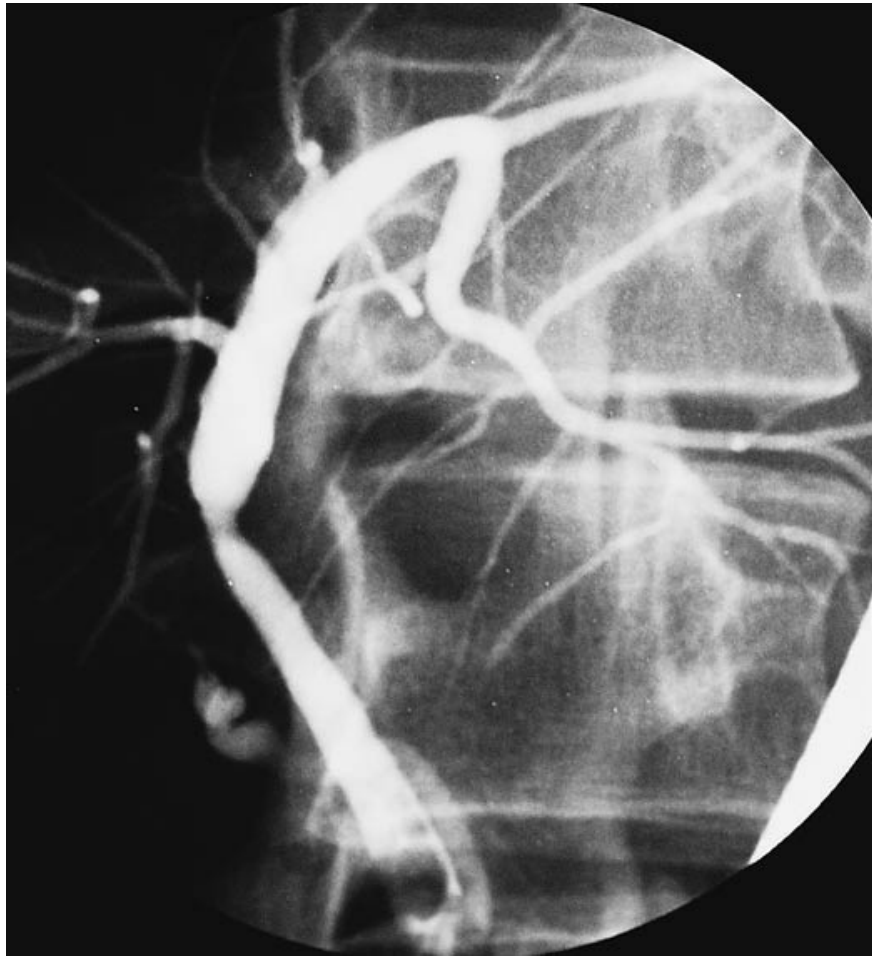

Figure 4 Endoscopic retrograde cholangiography (ERC) demonstrates the result of the treatment 4.5 years after the trauma. There is a stricture that is functionally irrelevant (the diameter of the bile duct is $3 \mathrm{~mm}$ ), with a good outflow of contrast medium into the duodenum. The intrahepatic bile ducts are not dilated.

References

${ }^{1}$ Prat F, Pelletier G, Ponchon T et al. What role can endoscopy play in the management of biliary complications after laparoscopic cholecystectomy? Endoscopy 1997; 29: $341-348$

2 Neuhaus P, Schmidt SC, Hintze RE et al. Classification and treatment of bile duct lesions following laparoscopic cholecystectomy. Chirurg 2000; 71: 166-173

${ }^{3}$ Lillemoe KD, Melton GB, Cameron JL et al. Postoperative bile duct strictures: Management and outcome in the 1990s. Ann Surg 2000; 232: 430 - 441

${ }^{4}$ Costamagna G, Pandolfi M, Mutignani $\mathrm{M}$ et al. Long-term results of endoscopic management of postoperative bile duct strictures with increasing numbers of stents. Gastrointest Endosc 2001; 154: $162-168$

${ }^{5}$ Veltzke-Schlieker W, Abou-Rebyeh $\mathrm{H}$, Adler A et al. Ergebnisse der Parallelstent-Applikation zur Therapie narbiger DHC-Stenosen. Z Gastroenterol 2002; 40: 693
Corresponding Author

\section{S. C. Schmidt, M.D.}

Department of General, Visceraland Transplantation Surgery Charité Campus Virchow Clinic University Medicine Berlin, Germany Augustenburger Platz 1 13353 Berlin

Fax:

+49-30-450552900

E-mail:

sven.schmidt@charite.de 